\title{
Evaluation of Parametrial Status in Locally Advanced Cervical Cancer Patients after Neoadjuvant Chemotherapy: A Prospective Study on Diagnostic Accuracy of Three-Dimensional Transvaginal Ultrasound
}

\author{
Giorgia Perniola Margherita Fischetti Federica Tomao Violante Di Donato \\ Innocenza Palaia Giusi Santangelo Francesca Lecce Maria Grazia Piccioni \\ Ludovico Muzii Pierluigi Benedetti Panici
}

Department of Maternal and Child Health and Urological Sciences, University "Sapienza", Policlinico "Umberto I", Rome, Italy

\section{Keywords}

Cervical cancer · Parametrial infiltration - Three-dimensional ultrasound $\cdot$ Magnetic resonance $\cdot$ Neoadjuvant

chemotherapy · Histology

\begin{abstract}
Objectives: To analyze the diagnostic accuracy of two-dimensional (2D) and three-dimensional transvaginal ultrasound (3D TV-US) for evaluation of parametrial status in locally advanced cervical cancer patients after neoadjuvant chemotherapy (NACT), using histology as the gold standard. Methods: Consecutive patients with histologically confirmed cervical cancer were staged according to FIGO (International Federation of Gynaecology and Obstetrics) criteria. All IB2-IIIB FIGO stage patients were examined by 2D and 3D TV-US and magnetic resonance imaging (MRI) at the diagnosis time (TO) and after NACT. At T0, the US evaluation of parametrial involvement was compared to MRI before treatment. The results of US and MRI examinations of parametrial status after NACT were compared with the histological specimen.
\end{abstract}

Results: We enroled 51 consecutive patients in the study. Before chemotherapy, clinical examination under anaesthesia identified parametrial involvement in 48 patients, ultrasonography in 46 patients, and MRI in 49 patients. The agreement between US and MRI was $94 \%$. The sensitivity of US for parametrial status was $93.8 \%$, with a positive predictive value of $97.8 \%$, using MRI as the standard. The correlation between US and MRI was statistically significant $(p=0)$. After chemotherapy, histological examination of surgical specimens identified parametrial involvement in 3 patients. Ultrasonography correctly identified those cases with parametrial infiltration, recording a sensitivity of $100 \%$, specificity of $90.9 \%$, positive predictive value of $50 \%$, and negative predictive value of $100 \%$. The MRI had a sensitivity of $100 \%$, specificity of $45.5 \%$, positive predictive value of $14.3 \%$, and negative predictive value of $100 \%$, respectively. The concordance in the identification of the presence/absence of infiltration between US and MRI with histology was $90 \%(p=0.001)$ and $61 \%$, respectively, after chemotherapy treatment. Particularly, in defining the degree of infiltration, the agreement between US and MRI with histology was 90 and 58\%, respec-

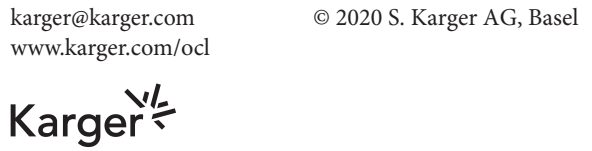

Dr. Margherita Fischetti

Department of Maternal and Child Health and Urological Sciences

University "Sapienza", Policlinico "Umberto I"

Viale del Policlinico 155, IT-00155 Rome (Italy)

margherita.fischetti@gmail.com 
tively. Conclusion: In locally advanced cervical cancer patients, 2D/3D TV-US can be considered accurate in the evaluation of parametrial infiltration to assess the response to NACT. It could be included as a diagnostic method in the preoperative work-up of cervical cancer.

(C) 2020 S. Karger AG, Basel

\section{Introduction}

Therapeutic management of uterine cervical cancer depends mainly on the FIGO (International Federation of Gynaecology and Obstetrics) clinical stage, even if its accuracy turned out to be suboptimal when compared with surgical and pathological data $[1,2]$. The extent of the disease is underestimated in about one third of women with early stage disease and up to two thirds of women with advanced disease $[1,2]$. Particularly craniocaudal extension of tumour volume and parametrial involvement are parameters that are not easy to estimate during the clinical examination. On the other hand, it is fundamental to know the state of these parameters to choose the best therapeutic management: neoadjuvant chemotherapy (NACT), radical surgery, and radiotherapy.

Because of discrepancies between clinical staging and pathological findings, the introduction of magnetic resonance imaging (MRI) for measuring tumour volume and local extent was suggested [3-8]. Computed tomography (CT) and positron emission tomography (PET) were introduced for the evaluation of metastatic disease $[9,10]$. However, the FIGO Gynaecologic Cancer Committee has accepted these sophisticated methods only reluctantly, because MRI, CT, and PET/CT are not universally available [3-10].

Fig. 1. a, b Tumour invasion at 3D US.
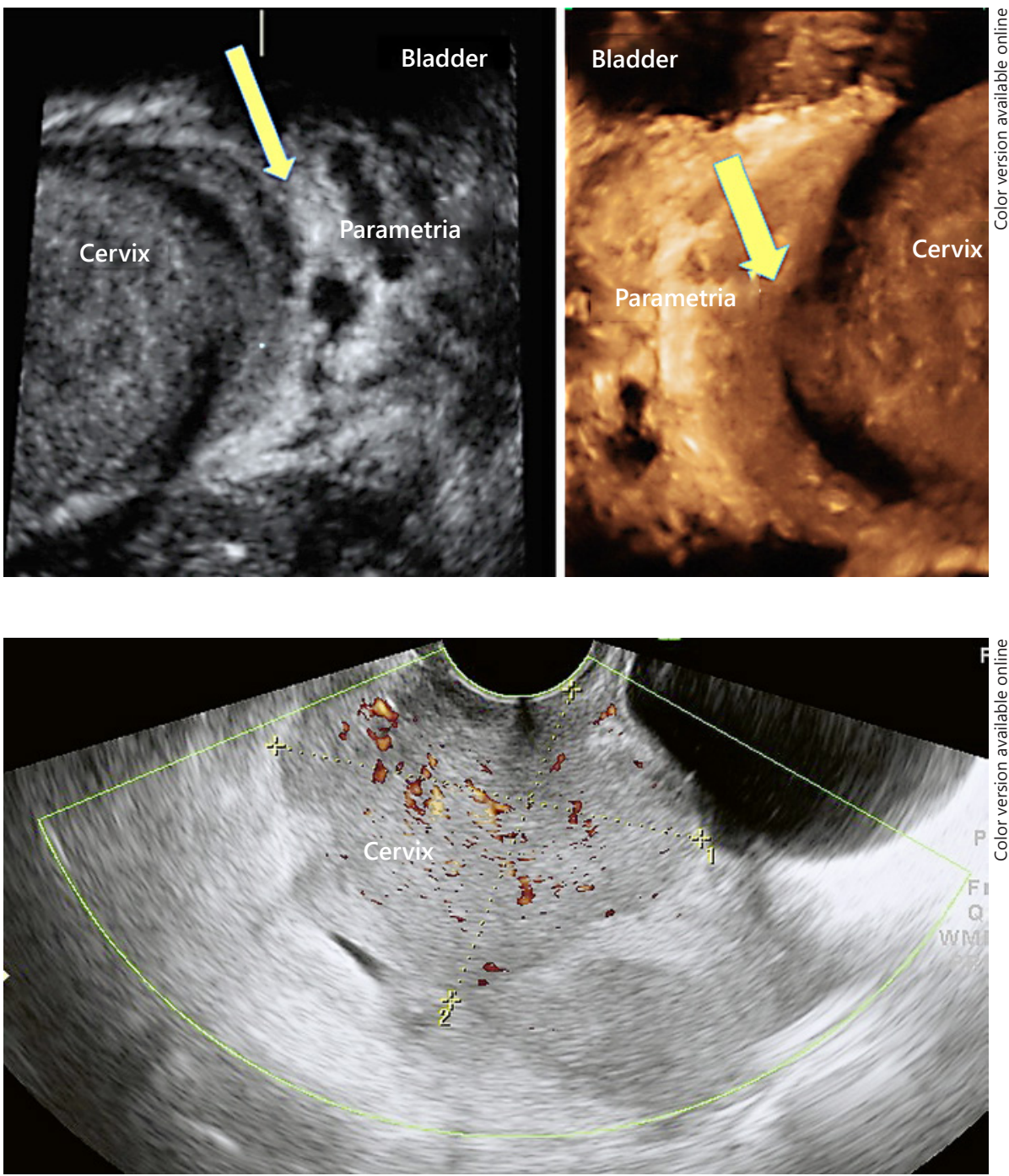

Fig. 2. Cervical cancer at US examination. 
In recent years ultrasound (US) has gained increased attention in preoperative staging of cervical cancer [11, 12 ]. US is faster, cheaper, more widely available, and requires no preparation of the patient compared to MRI. It is reasonable to consider US as a potential diagnostic tool to investigate a local spread of cervical cancer disease. Several studies showed that transvaginal and transrectal US are accurate and the results are comparable to MRI in the evaluation of the local extension of the disease [1113]. Up to now there has been a lack of data concerning the accuracy of US after NACT. In fact, it is hard to distinguish fibrosis from residual tumour, and the chemotherapy response is important to assess if the patient can undergo surgery or other treatments.

The main objective of this prospective study was to analyze the diagnostic accuracy of two-dimensional (2D)/ three-dimensional (3D) transvaginal US (TV-US) for the evaluation of parametrial infiltration in locally advanced cervical cancer patients after neoadjuvant chemotherapy, at this particular stage of the treatment, using histology as the standard. Our hypothesis is that $2 \mathrm{D} / 3 \mathrm{D}$ TV-US is accurate and comparable to MRI in the evaluation of the local extension of the cervical cancer.

The second objective was to assess the agreement between 3D US and MRI in evaluating parametrial involvement.

\section{Patients and Methods}

This prospective study included all women who were referred to our centre for histologically confirmed cervical cancer from September 2016 to December 2018. The local ethics committee approved the study, and all participating patients gave written informed consent.

All the patients were staged according to the FIGO criteria and underwent a vaginal and rectal examination under anaesthesia and a standard chest X-ray. Cystoscopy and sigmoidoscopy were performed when indicated.

Inclusion criteria were: IB2-IIIB FIGO stage, histologically confirmed cervical cancer, performance status $<3$ (ECOG classification). Exclusion criteria were: IA-IB1 and IV FIGO stage, performance status $>3$, or non-compliance of patients. All IB2-IIIB FIGO-staged patients underwent NACT. All patients who showed complete or partial response to treatment, according to the response evaluation of RECIST criteria in solid tumours [14], underwent surgery. All patients underwent 2D/3D TV-US and MRI within 1 week before NACT (T0) and 3 weeks after the last cycle of chemotherapy, within 1 week before surgery (T1).

Initially,2D US was performed, and later, with the 3D method, the tumour volume was obtained and coronary plane parameters were studied. Furthermore offline analysis of 3D US volumes can be used to define more precisely the location and degree of tumour invasion (Fig. 1a, b).

3D US for Evaluation of Parametrial Status in Cervical Cancer after NACT
The MRI and US examiner had access to the patient history, but they were blinded to the FIGO staging and the results of the other imaging techniques.

Both the US examiner and the examiner of the MR images were expert examiners and they assessed the following parameters and completed a dedicated form on paper: tumour diameters (craniocaudal length, and transverse and antero-posterior diameters), involvement of vagina, parametria, vesicovaginal and rectovaginal septum.

The US evaluation of parametrial involvement was compared to MRI at T0. The results of US and MRI before surgery (T1) were compared to the histological findings of the surgical specimens for the evaluation of parametrial infiltration after NACT. All patients received a PET/CT scan for the detection of any distant metastases before NACT. They repeated the scan after NACT if it was positive for distant metastases.

\section{US Examination}

TV-US was performed by the same sonographer, skilled in gynaecologic oncology, with at least 15 years of experience in the US evaluation of gynaecologic tumours. An US machine GE Voluson with a multifrequency of 5-9 MHz endovaginal transducer was used.

As already described, at US examination, cervical cancer appears as a solid lesion with a mostly hypoechoic echostructure in comparison to the surrounding cervical stroma [12] (Fig. 2). Pow-
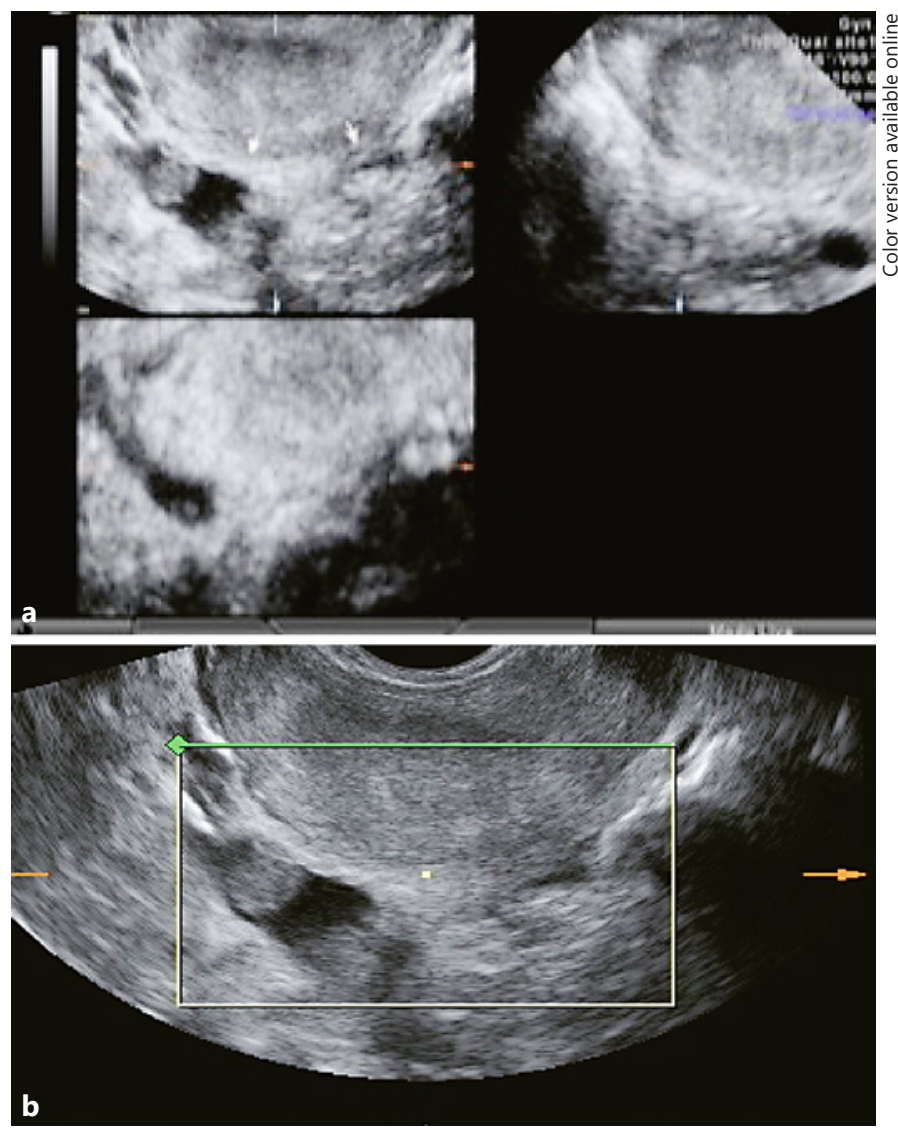

Fig. 3. a, b Mild parametrial infiltration at TV-US.

Oncology 2020;98:603-611 

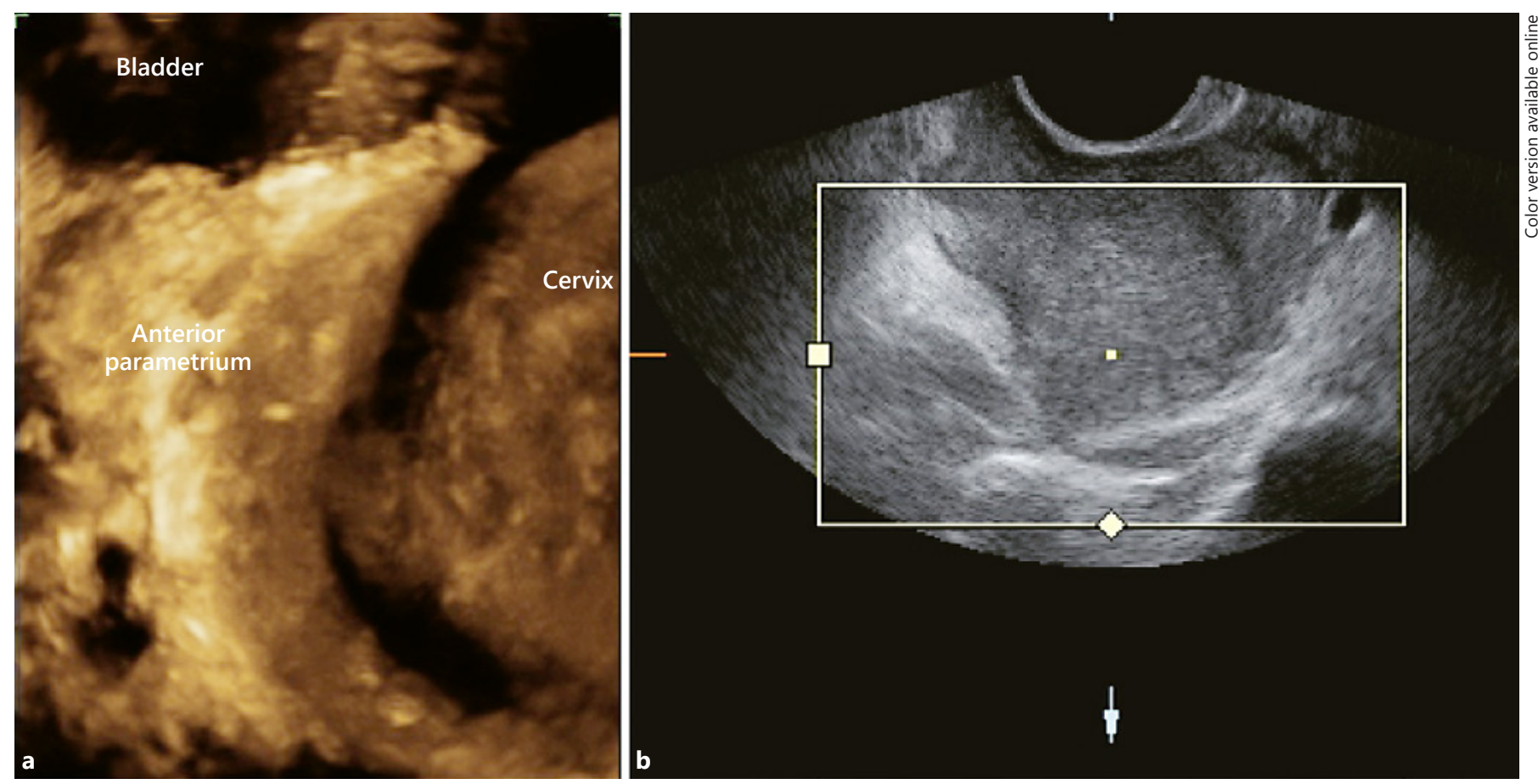

Fig. 4. a, b Moderate anterior parametrial infiltration at 3D US examination.

er Doppler ultrasonography is routinely included in our sonographic examinations.

Sonographic findings suggestive of a parametrial invasion are an extension of the cervical tumour beyond the cervical stroma. A mild parametrial involvement, defined as grade 1 [11], was assessed as the presence of hypoechoic irregular spiculae infiltrating the pericervical tissue (Fig. 3a, b). A moderate parametrial involvement, defined as grade 2 [11], was assessed as an infiltration from the cervical stroma in a lobular shape (Fig. 4a, b). A severe parametrial involvement, defined as grade 3 [11], was assessed as a complete parametrial infiltration (Fig. 5): anteriorly up to the bladder, laterally up to the external iliac vessels, and posteriorly up to the rectum. Infiltration of the vesicovaginal septum was diagnosed on the findings of a direct tumour extension and a lack of sliding of the posterior bladder wall from the uterus and the vagina. Infiltration of rectovaginal septum was assessed as the presence of direct tumour extension and a lack of sliding of the uterus and the vagina from the anterior rectal wall. The kidneys and ureters were always checked.

The results were immediately recorded on a dedicated form on paper to be used for subsequent statistical analysis and US photographic images were saved and stored on a hard disk.

The 3D grey-scale US volume was obtained using the maximum volume angle $\left(75^{\circ}\right)$, and it includes the uterine cervix, the bladder partially, and the rectum partially. 3D power Doppler volumes were obtained using a $75^{\circ}$ volume angle, a pulse repetition frequency of $0.6 \mathrm{kHz}$ and an image angle including the cervix and pericervical tissues.

Each volume of parameters was taken with a longitudinal scan for the anterior section, left and right, respectively, and with a single transverse scan for the posterior and lateral section.
Using the "sectional planes" modality, we examined the cervix with its craniocaudal axis longitudinal. The parametrial involvement was examined by scrolling through the cervix showing a transverse plane through the cervix. The transverse planes of the cervix were analyzed with regard to parametrial infiltration as present or absent, and if present the extent of the infiltration was graded as described above.

\section{MRI Examination}

MRI was performed in all cases with a 3 T GE Discovery 750. To achieve a reduction of intestinal peristalsis in order to avoid motion artefacts, a hypotonic drug was given intravenously (hyoscine N-butylbromide, Buscopan $20 \mathrm{mg} / \mathrm{ml}$, Boehringer Ingelheim $20 \mathrm{mg}$ ). The bladder was full.

The patient was introduced into the gantry, on her back, "feet first" with a multichannel coil positioned over the lower abdomen (8-channel $127.73 \mathrm{MHz}$ ).

The following sequences were studied:

- Single shot fast spin echo;

- T2 w FRFSE HR with para-axial, sagittal and paracoronal acquisitions;

- DWI b 0-1,000 with ADC map;

- T1 ESF w;

- Lava-flex pre- and post-contrastographic.

The following parameters evaluated:

- Recognition and size parameters of the tumour lesion in T2weighted acquisitions;

- Signal diffusion (DWI) and ADC value;

- Infiltration of the parameters: a mild parametrial involvement, defined as grade 1 , was assessed as the presence of infiltration of the pericervical tissue. A moderate parametrial involvement, 
Fig. 5. Severe anterior parametrial infiltration at $3 \mathrm{D}$ US examination.

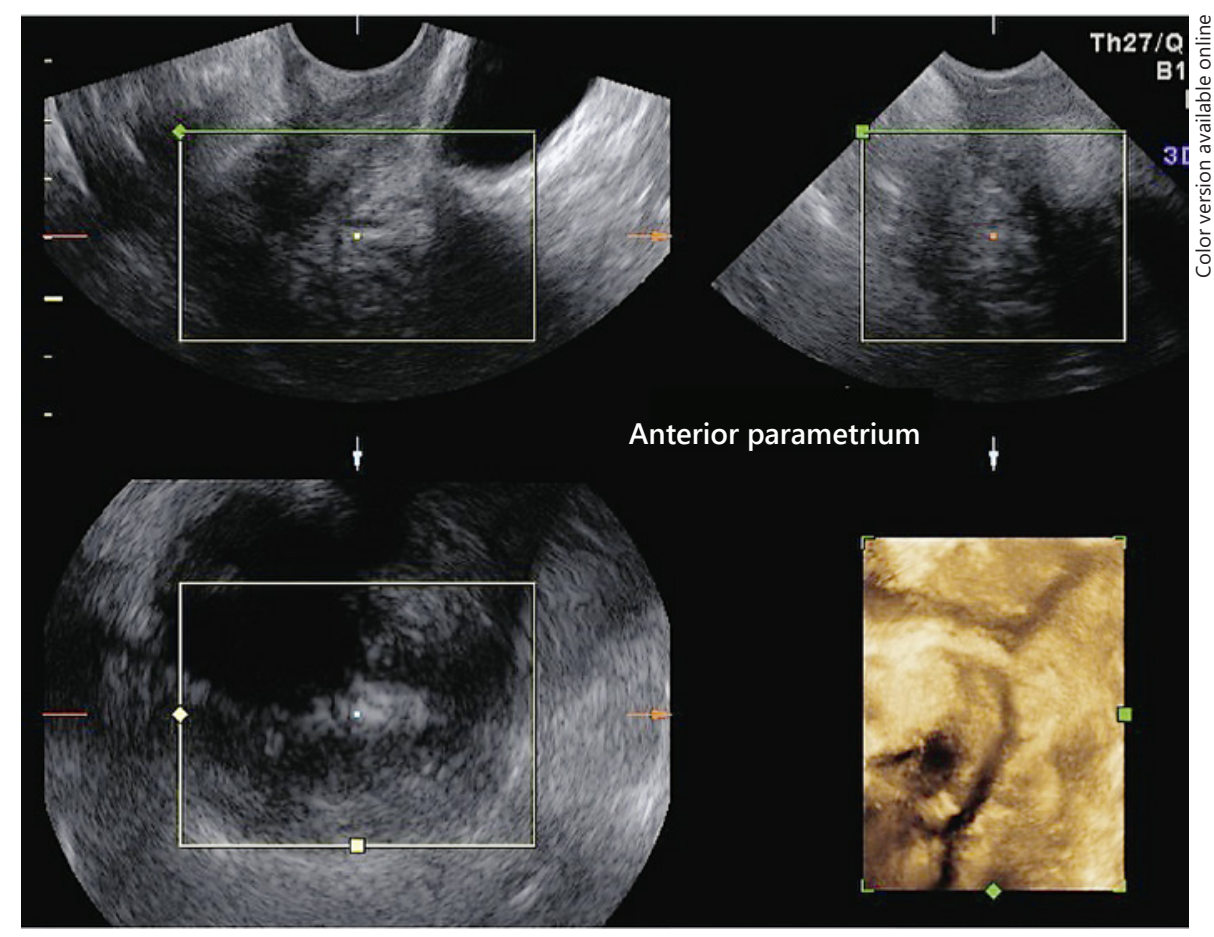

Table 1. Characteristics of patients

\begin{tabular}{lc}
\hline Patients, $N$ & 51 \\
Age (mean), years & $55.3 \pm 13.4$ \\
Histotype, $n(\%)$ & \\
$\quad$ Adenocarcinoma & $10(20)$ \\
Squamous & $41(80)$ \\
FIGO stage, $n \%)$ & \\
$\quad$ IB2 & $3(6)$ \\
IIB & $26(51)$ \\
IIIA & $1(2)$ \\
IIIB & $21(41)$ \\
Grading, $n(\%)$ & \\
G1 & $4(7.8)$ \\
G2 & $25(49)$ \\
G3 & $22(43.2)$ \\
\hline
\end{tabular}

defined as grade 2 , was assessed as an infiltration from the cervical stroma. A severe parametrial involvement, defined as grade 3 , was assessed as a complete parametrial infiltration;

- Longitudinal extent;

- Vaginal involvement;

- Infiltration of the bladder and/or rectal wall.

\section{Statistics}

The effectiveness of 2D/3D US to assess local regional staging of cervix tumour was evaluated before and after NACT. 2D/3D US diagnostic accuracy, specificity, sensitivity, and positive and negative predictive values were calculated, setting MRI and pathological findings as the standard, respectively, before and after NACT.

3D US for Evaluation of Parametrial Status in Cervical Cancer after NACT
Partial correlation was defined if any grade of parametrial infiltration was found by the two techniques (e.g., positive parametrium: 3D US and MRI or negative parametrium: 3D US and MRI). Complete correlation was defined if the same grade of parametrial infiltration was found by the two techniques (G1 positive parametrium; G2 positive parametrium; G3 positive parametrium; negative parametrium).

The incidence of events among the groups was analyzed for statistical significance using Fisher's exact test. The $t$-test and Mann-Whitney $U$ test were used to compare variables with and without Gaussian distribution, respectively. A $p$ value $<0.05$ was considered statistically significant. Statistical analysis was performed with GraphPad Prism version 6.0 for Mac (GraphPad Software, San Diego, CA, USA) and IBM-Microsoft SPSS version 22.0 for Mac.

\section{Results}

Sixty-seven consecutive patients were enroled from September 2016 to December 2018 with a histologically confirmed diagnosis of cervical cancer. Sixteen patients were excluded from the study because they presented an early stage at clinical and instrumental examination (MRI and US) and they were sent to radical hysterectomy. The histology examination confirmed that they were early cervical cancer cases. We included 51 patients in the study. Table 1 shows the characteristics of the patients. 
Table 2. Parametrial infiltration (G0, G1-G2, G3) before NACT: MRI versus examination under anaesthesia

\begin{tabular}{llcc}
\hline & \multicolumn{2}{l}{ MRI (T0) } \\
\cline { 2 - 4 } $\begin{array}{l}\text { Examination under } \\
\text { anaesthesia }\end{array}$ & G0 & G1-G2 & G3 \\
\hline G0 & & & \\
G1-G2 & $1(2 \%)$ & $1(2 \%)$ & $1(2 \%)$ \\
G3 & $1(2 \%)$ & $18(35 \%)$ & $8(16 \%)$ \\
& 0 & $10(20 \%)$ & $11(21 \%)$ \\
\hline
\end{tabular}

Table 4. Parametrial infiltration (G0, G1-G2, G3) before NACT: 3D TV-US versus MRI

\begin{tabular}{llrl}
\hline \multirow{2}{*}{ TV-US } & \multicolumn{2}{l}{ MRI } & \\
\cline { 2 - 4 } G0 & G1-G2 & G3 \\
\hline G0 & $2(4 \%)$ & $3(6 \%)$ & 0 \\
G1-G2 & 0 & $18(35 \%)$ & $5(10 \%)$ \\
G3 & 0 & $8(16 \%)$ & $15(29 \%)$ \\
\hline
\end{tabular}

Specifically, 3 (6\%) patients were staged as FIGO IB2, 26 (51\%) patients were staged IIB, 1 (2\%) patient was staged IIIA, and 21 (41\%) patients were staged IIIB. Histological examination showed that 41 patients $(80 \%)$ had a squamous carcinoma histotype, and $10(20 \%)$ patients had an adenocarcinoma. Three patients had a pelvic positive node at PET/CT.

After NACT $32(62 \%)$ patients had a partial clinical response, $6(12 \%)$ a complete clinical response, $4(7 \%)$ patients had stable disease, and 9 (19\%) patients had disease progression. These last 13 patients were referred to concomitant chemoradiotherapy and because there was no histological examination, we excluded them from the final analysis. Thirty-eight patients underwent radical surgery: 5 patients type B hysterectomy, 28 patients type C hysterectomy, and 5 patients type D hysterectomy.

All patients underwent both 2D/3D TV-US and MRI before and after NACT. At T0, vaginal and rectal examination under anaesthesia identified parametrial involvement in 48 patients, ultrasonography in 46 patients, and MRI in 49 patients.

For assessing the presence/absence of parametrial infiltration (partial correlation), the agreement between MRI and examination under anaesthesia was $94 \%$ (48/51 patients), with disagreement in 3 cases: in 1 patient the MRI was negative, while the examination under anaes-
Table 3. Parametrial infiltration (G0, G1-G2, G3) before NACT: 3D TV-US versus examination under anaesthesia

\begin{tabular}{llcc}
\hline & \multicolumn{3}{l}{ Examination under anaesthesia } \\
\cline { 2 - 4 } TV-US (T0) & G0 & G1-G2 & G3 \\
\hline G0 & $1(2 \%)$ & $4(8 \%)$ & 0 \\
G1-G2 & $2(4 \%)$ & $18(35 \%)$ & $3(6 \%)$ \\
G3 & 0 & $5(10 \%)$ & $18(35 \%)$ \\
\hline
\end{tabular}

Table 5. Parametrial infiltration (G0, G1-G2, G3) after NACT: 3D TV-US versus histology

\begin{tabular}{llll}
\hline & \multicolumn{2}{l}{ Histology } & \\
\cline { 2 - 4 } TV-US (T1) & G0 & G1-G2 & G3 \\
\hline G0 & $31(82 \%)$ & 0 & 0 \\
G1-G2 & $4(10 \%)$ & $3(8 \%)$ & 0 \\
G3 & 0 & 0 & 0 \\
\hline
\end{tabular}

thesia found parametrial involvement. In 2 cases, MRI was positive while examination under anaesthesia was negative (Table 2). The agreement between 3D US and examination under anaesthesia was $88 \%$ (45/51 patients). In 4 patients US was negative and examination under anaesthesia showed grade G1-2 infiltration and in 2 cases at US parametrial involvement was present and examination under anaesthesia was negative (Table 3 ).

The agreement between 3D US and MRI was $94 \%$ (48/51 patients) with disagreement in 5cases: in 3 cases, 3D US was negative and MRI suggested that there was infiltration (Table 4).

For assessing the grade of parametrial infiltration (total correlation), the examination under anaesthesia agreed with MRI in 57\% of cases (29/51 patients): in 18 patients the examination agreed for G1-2 infiltration and in 11 patients it agreed for G3 infiltration, 10 patients had grade G1-2 infiltration with MRI and grade G3 with the examination under anaesthesia, 8 patients had grade G3 infiltration with MRI and grade G1-2 with the examination under anaesthesia (Table 2). The US agreed with MRI in $65 \%$ of cases (33/51): in 18 patients it agreed for G1-2 infiltration and in 15 patients it agreed for G3 infiltration, 8 patients had grade G1-2 infiltration with MRI and grade G3 with 3D US, 5 patients had grade G3 infiltration with MRI and grade G1-2 with 3D US (Table 4). 
Table 6. Parametrial infiltration (G0, G1-G2, G3) after NACT: MRI versus histology

\begin{tabular}{llll}
\hline & \multicolumn{2}{l}{ Histology } & \\
\cline { 2 - 4 } MRI (T1) & G0 & G1-G2 & G3 \\
\hline G0 & $20(53 \%)$ & 0 & 0 \\
G1-G2 & $15(39 \%)$ & $2(5 \%)$ & 0 \\
G3 & 0 & $1(3 \%)$ & 0 \\
\hline
\end{tabular}

Table 7. Parametrial infiltration (G0, G1-G2, G3) after NACT: $3 D$ TV-US and MRI in 51 patients (all parametria)

\begin{tabular}{llll}
\hline & \multicolumn{2}{l}{ MRI (T1) } & \\
\cline { 2 - 4 } TV-US & G0 & G1-G2 & G3 \\
\hline G0 & $17(33 \%)$ & $15(29 \%)$ & 0 \\
G1-G2 & $3(6 \%)$ & $8(16 \%)$ & $8(16 \%)$ \\
G3 & 0 & 0 & 0 \\
\hline
\end{tabular}

The sensitivity of 3D US parametrial infiltration was $93.8 \%$, with a positive predictive value of $97.8 \%$, using MRI as the standard. The correlation between US and MRI was statistically significant $(p<0.0001)$.

Histological findings of surgical specimens identified parametrial involvement in 3 patients.

Ultrasonography and MRI showed the following results: the sensitivity was $100 \%$ both for US that for MRI, specificity 90.9 and $45.5 \%$, respectively, positive predictive value 50 and $14.3 \%$, respectively, and negative predictive value $100 \%$ both for US and for MRI.

The diagnostic performance of US and MRI in comparison to histology as the gold standard is shown in Tables 5 and 6 . The concordance between US and histology was $90 \%$ and statistically significant $(p=0.001)$ both in the identification of the presence/absence of infiltration after the chemotherapy treatment (partial correlation), and in defining the degree of infiltration (total correlation). In 4 cases (10\%) 3D US showed grade G1-2 infiltration and histology showed absence of infiltration (Table 5). The agreement, however, between MRI and histology was $61 \%$ (23/38 patients) in the identification of the presence/absence of parametrial infiltration with disagreement in 15 cases: in these cases the histology was negative, while MRI showed grade G1-2 infiltration (Table 6 ). The total correlation was $58 \%$ (22/38 patients): in
20 patients for absence of infiltration and in 2 patients for G1-2 infiltration, 1 patient had grade G1-2 infiltration at histology and grade G3 with MRI (Table 6).

Table 7 shows the concordance between 3D US and MRI after NACT in the identification of parametria involvement.

\section{Discussion}

In this study, comparing TV-US and MRI in the evaluation of parametrial status in locally advanced cervical cancer after NACT, we found that US has a sensitivity of $100 \%$ equal to MRI and a specificity which is higher than MRI (90.9 vs. $45.5 \%$ ). Particularly the agreement between US and MRI was 65\% in identifying the presence or absence of parametrial infiltration and was $49 \%$ in the evaluation of the degree of infiltration. To the best of our knowledge, this is the first study to evaluate the diagnostic accuracy of MRI and 3D US in the diagnosis of parametrial infiltration after NACT in patients with locally advanced cervical cancer, using histology as the standard.

The concordance between US and histology was $90 \%$ and statistically significant both in the identification of the presence/absence of infiltration after the chemotherapy treatment and in defining the degree of infiltration. There are 4 false-positive cases (10\%) at 3D US. The agreement between MRI and histology was $61 \%$ in the identification of the presence/absence of parametrial infiltration with 15 false-positive cases at MRI.

We would like to underline that after NACT it is particularly difficult to distinguish fibrosis from residual tumour. Fibrosis and necrosis change the normal structure of the pelvic organs and disrupt the borders between organs and structures; maybe with MRI it is more difficult to distinguish fibrosis from tumour presence. On the other hand, it is important to find the best imaging tool to evaluate the chemotherapy response in order to send patients for radical surgery or other treatments.

These patients are difficult to examine with any imaging method. In fact, in a recent study where Fischerova et al. [11] analyzed parametrial involvement without previous chemotherapy, they reported better results both with US and MRI. Specifically, the accuracy of US compared to MRI was 98.9 versus $94.7 \%$ in the evaluation of the parametrial infiltration.

Additional previous studies confirmed our results of how TV-US can detect cervical cancer spread with good specificity. Testa et al. [12] showed that 2D TV-US and 
MRI have a similar sensitivity in the evaluation of parametrial infiltration (60 and 40\%, respectively). Recently, a study has been published on the correlation between the accuracy of 2D and 3D US and MRI on parametrial infiltration, showing a high correlation (79\%), similar to our own results (84\%) [15]. In another recent study, 3D sonography showed good agreement with MRI for assessing parametrial infiltration and bladder involvement [16]. These studies could not use histology as the gold standard to evaluate the real parametrial infiltration.

The use of MRI as the standard for the evaluation of parametrial involvement can be questioned after the recently cited published data. The detection rate of parametrial infiltration by MRI was only $50 \%$ with a specificity of $98 \%$ in one study and only $40 \%$ with a specificity of $89 \%$ in another study when histology was used as reference $[11,12]$.

The discrepancy between US and MRI might be explained by 3D US allowing continuous scrolling through the cervix while the analysis with MRI is limited by slice thickness.

In our study, before chemotherapy, the correlation between examination under anaesthesia and MRI also was 94\% in the diagnosis of the presence/absence of parametrial invasion but was lower in the evaluation of the degree of infiltration (57\%), while the examination under anaesthesia compared with US had an agreement of $88 \%$. Therefore, US compared with MRI is as accurate as the examination under anaesthesia to identify parametrial infiltration, and it is more accurate in identifying the degree of infiltration.

Therefore, it is not at all certain whether the disagreement of US and MRI in the evaluation of parametrial involvement in our study was due to an underestimation by US. We had disagreement in 15 cases: in 15 (29\%) cases the 3D US was negative and MRI suggested infiltration (grade 1-2); in 3 cases 3D US showed grade 1-2 parametrial infiltration while MRI was negative.

The 3D US has several advantages over 2D US and transrectal US such as the offline analysis allowing the US examiner to review in detail the stored volumes at any time and as many times as needed and without the patient being present. Stored volumes are also suitable for a second opinion. Moreover, when using 3D US, the location of parametrial infiltration can be estimated more precisely than with 2D US. This could be important when planning tailored nerve-sparing surgery. We believe that $2 \mathrm{D}$ and 3D US should be regarded as complementary techniques. 2D dynamic US can be used routinely to detect the presence of infiltration, while offline analysis of $3 \mathrm{D}$ US volumes can be used to define more precisely the location and degree of tumour invasion. However, we believe $3 \mathrm{D}$ US examination is complementary to $2 \mathrm{D}$ US, which retains its dynamic nature as the movement of the transvaginal probe allows the operator to examine the flow of contiguous tissue against the probe, facilitating the evaluation of a tumour.

The disadvantages of MRI are the cost, the time needed for the execution of the procedure, the static nature of the examination, and the presence of possible contraindications (pacemakers, claustrophobia).

It is a strength that our study is prospective, that both radiologists and US examiners used the same standardized research protocol, and that they analyzed parametrial infiltration by assessing the same planes (coronal plane) through the cervix. Moreover, radiologists and US examiners did not only record parametrial infiltration as present or absent, but also assessed the localization and the extent (grade) of infiltration using the same terminology. The knowledge of the extent of parametrial involvement is particularly helpful for the surgeons. In fact, the radicality of the surgery could be tailored according to imaging results.

Thus, the ability of transvaginal sonography to analyze the uterine cervix in these patients after NACT and to evaluate the chemotherapy response is of great relevance in supporting the validity of this technique.

Inter-observer reproducibility was not tested and we considered it a limitation in this study. The main weakness of our study is the limited size of the group of patients. However, despite this, we confirmed the statistically significant agreement between MRI and 3D US before chemotherapy and between 3D US and histology after chemotherapy.

\section{Conclusions}

In the hands of experienced specialists in gynaecological oncology, US can be considered as accurate an examination as MRI in the evaluation of parametrial infiltration in patients with locally advanced cervical cancer. US provides an optimal staging and a corrected evaluation of the chemotherapy response of these patients. Considering the relatively low cost, wide availability, rapidity of execution, and the possibility of a dynamic examination, 3D US should be included as a diagnostic method in the preoperative work-up of cervical cancer above all after NACT. 


\section{Statement of Ethics}

Our research is compliant with the guidelines for human studies and research was conducted ethically in accordance with the World Medical Association Declaration of Helsinki.

\section{Disclosure Statement}

The authors have no conflicts of interest to declare.

\section{Author Contributions}

All authors have made substantial contributions to all of the following: the conception and design, acquisition of data, drafting the article, revising it critically for important intellectual content, and final approval of the version to be submitted. Perniola Giorgia: conception and design, drafting the article. Margherita Fischetti: acquisition of data, drafting the article. Federica Tomao: drafting the article. Violante Di Donato: acquisition of data. Maria GraziaPiccioni, Ludovico Muzii, and Pierluigi Benedetti Panici: revising the article critically for important intellectual content.

\section{Funding}

None.

\section{References}

1 Delgado G, Bundy B, Zaino R, Sevin BU, Creasman WT, Major F. Prospective surgicalpathological study of disease-free interval in patients with stage IB squamous cell carcinoma of the cervix: a Gynecologic Oncology Group study. Gynecol Oncol. 1990;38(3): 352-7.

2 Lagasse LD, Creasman WT, Shingleton HM, Ford JH, Blessing JA. Results and complications of operative staging in cervical cancer: experience of the Gynecologic Oncology Group. Gynecol Oncol. 1980;9(1):90-8.

3 Monk BJ, Tewari KS. Invasive cervical cancer. In: DiSaia PJ, Creasman WT (eds). Gynecologic oncology. Philadelphia: Elsevier Science; 2007. p. 54-124.

4 Odicino F, Pecorelli S, Zigliani L, Creasman WT. History of the FIGO cancer staging system. Int J Gynaecol Obstet. 2008;101(2):20510.

5 Van Nagell JR, Roddick JW Jr, Lowin DM. The staging of cervical cancer: inevitable discrepancies between clinical staging and pathologic findings. Am J Obstet Gynecol. 1971;110(7):973-8.

6 Lagasse LD, Creasman WT, Shingleton HM, Ford JH, Blessing JA. Results and complications of operative staging in cervical cancer: experience of the Gynecologic Oncology Group. Gynecol Oncol. 1980;9(1):90-8.
7 Delgado G, Bundy B, Zaino R, Sevin BU, Creasman WT, Major F. Prospective surgicalpathological study of disease-free interval in patients with stage IB squamous cell carcinoma of the cervix: a Gynecologic Oncology Group study. Gynecol Oncol. 1990;38(3): 352-7.

8 Zander J, Baltzer J, Lohe KJ, Ober KG, Kaufmann C. Carcinoma of the cervix: an attempt to individualize treatment. Results of a 20 -year cooperative study. Am J Obstet Gynecol. 1981;139(7):752-9.

9 Hricak H, Yu KK. Radiology in invasive cervical cancer. AJR Am J Roentgenol. 1996; 167(5):1101-8

10 Trimble EL. Cervical cancer state-of-the-clinical-science meeting on pretreatment evaluation and prognostic factors, September 2728, 2007: proceedings and recommendations. Gynecol Oncol. 2009;114(2):145-50.

11 Fischerova D, Cibula D, Stenhova H, Vondrichova H, Calda P, Zikan M, et al. Transrectal ultrasound and magnetic resonance imaging in staging of early cervical cancer. Int J Gynecol Cancer. 2008;18(4):766-72.
12 Testa AC, Ludovisi M, Manfredi R, Zannoni G, Gui B, Basso D, et al. Transvaginal ultrasonography and magnetic resonance imaging for assessment of presence, size and extent of invasive cervical cancer. Ultrasound Obstet Gynecol. 2009;34(3):335-44.

13 Epstein E, Testa A, Gaurilcikas A, Di Legge A, Ameye L, Atstupenaite V, et al. Early-stage cervical cancer: tumor delineation by magnetic resonance imaging and ultrasound-a European multicenter trial. Gynecol Oncol. 2013;128(3):449-53.

14 Eisenhauer EA, Therasse P, Bogaerts J, Schwartz LH, Sargent D, Ford R, et al. New response evaluation criteria in solid tumours: revised RECIST guideline (version 1.1). Eur J Cancer. 2009;45(2):228-47.

15 Chiappa V, Di Legge A, Valentini AL, Gui B, Miccò M, Ludovisi M, et al. Agreement of two-dimensional and three-dimensional transvaginal ultrasound with magnetic resonance imaging in assessment of parametrial infiltration in cervical cancer. Ultrasound Obstet Gynecol. 2015 Apr;45(4):459-69.

16 Arribas S, Alcázar JL, Arraiza M, Benito A, Minguez JA, Jurado M. Three-dimensional transvaginal sonography and magnetic resonance imaging for local staging of cervical cancer: an agreement study. J Ultrasound Med. 2016 May;35(5):867-73. 